\title{
Primordial follicle activation as new treatment for primary ovarian insufficiency
}

\author{
Hye Nam Lee, Eun Mi Chang \\ Department of Obstetrics and Gynecology, Fertility Center, CHA Gangnam Medical Center, CHA University, Seoul, Korea
}

Primordial follicle activation is a process in which individual primordial follicles leave their dormant state and enter a growth phase. While existing hormone stimulation strategies targeted the growing follicles, the remaining dormant primordial follicles were ruled out from clinical use. Recently, in vitro activation (IVA), which is a method for controlling primordial follicle activation, has provided an innovative technology for primary ovarian insufficiency (POI) patients. IVA was developed based on Hippo signaling and phosphatase and tensin homolog (PTEN)/phosphatidylinositol-3-kinase (PI3K)/protein kinase B (AKT)/forkhead box O3 (FOXO3) signaling modulation. With this method, dormant primordial follicles are activated to enter growth phase and developed into competent oocytes. IVA has been successfully applied in POI patients who only have a few remaining remnant primordial follicles in the ovary, and healthy pregnancies and deliveries have been reported. IVA may also provide a promising option for fertility preservation in cancer patients and prepubertal girls whose fertility preservation choices are limited to tissue cryopreservation. Here, we review the basic mechanisms, translational studies, and current clinical results for IVA. Limitations and further study requirements that could potentially optimize IVA for future use will also be discussed.

Keywords: Fertility preservation; Primary ovarian insufficiency; Primordial follicle activation; Ovarian follicle

\section{Introduction}

Primary ovarian insufficiency (POI) is diagnosed based on the cessation of menses in women younger than 40 years of age. Since its first report in 1942 by Fuller Albright, this condition has been heterogeneously termed premature ovarian failure, early menopause, and premature menopause. In POI, ovarian follicles decrease below a certain threshold and lose the potential for ovulation, leading to amenorrhea. The incidence of POI has been reported to be $1 \%$ in women younger than age 40 and $0.1 \%$ among women under 30 [1-3]. Proper

Received: Apr 1, 2019 · Revised: Apr 20, 2019 Accepted: May 16, 2019 Corresponding author: Eun Mi Chang

Department of Obstetrics and Gynecology, Fertility Center, CHA Gangnam Medical Center, CHA University, 566 Nonhyeon-ro, Gangnam-gu, Seoul 06135, Korea

Tel: +82-2-3468-3410 Fax:+82-2-3468-3464 E mail: emchang@cha.ac.kr

* This work was supported by grant from National Research Foundation of Korea (NRF-2017R1A1A1A05001385).

This is an Open Access article distributed under the terms of the Creative Commons Attribution Non-Commercial License (http://creativecommons.org/licenses/by-nc/4.0/) which permits unrestricted non-commercial use, distribution, and reproduction in any medium, provided the original work is properly cited. diagnostic criteria for POI are currently lacking; however, oligomenorrhea or amenorrhea for at least 4 months and persistently elevated follicle-stimulating hormone levels that exceed $25-40 \mathrm{IU} / \mathrm{L}$ on two occasions more than 4 weeks apart in women under the age of 40 defines POI clinically [4]. The cause of POI is mostly idiopathic, although genetic factors, including permutations in FMR1, X-chromosome deletion abnormalities, and single gene mutation, have been identified [5]. Additionally, autoimmune disorders, such as autoimmune polyglandular syndrome, and iatrogenic factors, including chemotherapy, radiation therapy, and ovarian surgery, are other known causes [6,7]. Because patients with POI do not respond to traditional fertility treatments, options for raising children for these patients have been adoption or egg donation, despite their continued hope for genetic offspring.

Three out of four women with POI have ovarian follicles remaining in the ovaries; yet, these follicles remain dormant. Thus, novel treatments to awaken dormant primordial follicles are urgently needed [1]. Pioneering studies have evaluated the use of gamete formation from stem cells, isolation of ovarian stem cells, activation of dormant follicles using in vitro activation (IVA), ovarian fragmentation for follic- 
ular activation (OFFA), stem cell ovarian transplantation, and injection of platelet-rich plasma. Among these methods, IVA has been successfully applied in humans with promising results. Here, we review IVA, a new potential treatment for POI patients.

\section{Basic and translational studies for primordial follicle activation}

\section{What maintains primordial follicle dormancy?}

Current hormonal stimulation strategies to induce multiple ovulations are only effective for the growing follicles, and the remaining dormant primordial follicles are therefore unable to be activated for potential clinical utility. While understanding the biological basis of primordial follicle activation is critical, the molecular mechanism underlying maintenance of either the dormant or activated states of primordial follicles is far from completely understood. It is known that multiple local factors and intracellular signaling pathways are involved. Multiple activators (BMP4/7, GDF-9, KIT-ligand, FGF2/7, insulin, GREM1/2, and LIF) and suppressors (AMH, LHX8, PTEN, Tsc1m/ TORC1, FOXO3a, YAP/Hippo signaling, and FOXL2) have been reported to be related to primordial follicle development [8-20]. Recently, focus has been placed on the phosphatase and tensin homolog (PTEN)/phosphatidylinositol-3-kinase (PI3K)/protein kinase B (AKT)/ forkhead box $\mathrm{O} 3$ (FOXO3) and Hippo signaling pathways.

\section{Primordial follicle activation mechanism \\ 1) PTEN/PI3K/AKT/FOXO3 signaling pathway}

The PTEN/PI3K/AKT/FOXO3 pathway has been implicated as a major signaling pathway involved in cancer; however, biochemical and genetic studies in mice have revealed that this pathway also plays an important role in the regulation of dormancy and initial follicular activation in ovary. FOXO subclasses are transcription factors that have important roles in cell cycle arrest, apoptosis, and stress responses in vitro [21]. While disruption of the FOXO1 gene results in embryonic lethality as a consequence of incomplete vascular development, deletion of the FOXO3a gene in a mouse model resulted in offspring that were viable and grossly indistinguishable from their littermate controls. FOXO3a-null females, however, showed age-dependent infertility and abnormal ovarian follicle development. These mice exhibited normal assembly of the primordial follicles followed by immediate global activation, resulting in ovarian hyperplasia, follicle depletion, premature ovarian failure, and infertility [22]. Within oocytes, FOXO3a is regulated by nucleocytoplasmic shuttling, essentially being imported into the nucleus during primordial follicle assembly and exported upon activation. Within the oocytes, FOXO3a may have a suppressive effect on initiation of follicular growth by affecting the mechanisms intrinsic to the ovary [21].

Oocyte-specific deletion of PTEN also caused global primordial follicle activation, similar to the phenotype induced by FOXO3a knock-

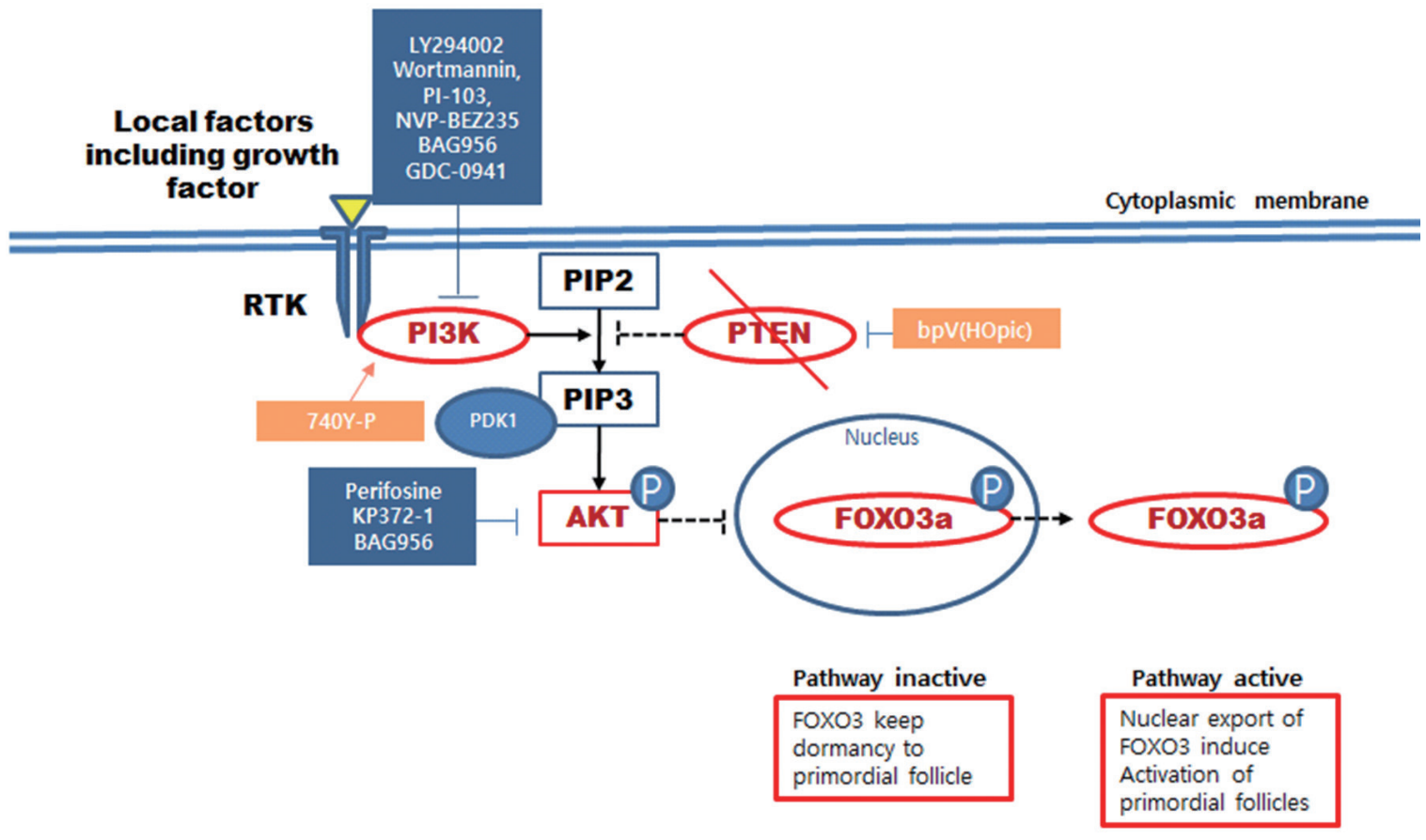

Figure 1. PTEN/PI3K/AKT/FOXO3 signaling and pathway modulators. RTK, receptor tyrosine kinase; PI3K, phosphatidylinositol-3-kinase; PIP2, phosphatidylinositol-4,5-bisphosphate; PIP3, phosphatidylinositol-3,4,5-trisphosphate; PDK1, phosphatidylinositol-dependent kinase 1; AKT, protein kinase B; P; phosphate group; FOXO3, forkhead box O3; PTEN, phosphatase and tensin homolog. 
out, and resulted in POI [23]. Interestingly, both the FOXO3- and the PTEN-knockout female mice are initially fertile, indicating that the deletion of either of the factors does not appear to disrupt subsequent steps of follicle maturation, ovulation, and fertilization. Another study utilizing pharmacological inhibition of PI3K showed that PVI3K suppressed the PTEN-knockout but not the FOXO3-knockout ovarian phenotype, suggesting that FOXO3 lies downstream of PTEN [24]. Furthermore, oocyte-specific ablation of PTEN resulted in PI3Kinduced conversion of secondary messenger phosphatidylinositol-4, 5-bisphosphate (PIP2) into phosphatidylinositol-3,4,5-trisphosphate (PIP3), which then activates phosphatidylinositol-dependent kinase 1 (PDK1), resulting in AKT activation [24]. Within the nucleus, AKT activation leads to FOXO3a hyperphosphorylation and nuclear export, thereby triggering primordial follicle activation. These findings suggest that hypoactivation or negative regulation of the PTEN/PI3K/ AKT/FOXO3 pathway may cause retardation of follicle activation and excessive primordial follicle atresia. On the contrary, inhibition of PTEN and activation of PI3K may cause activation of dormant follicles, since PTEN is a negative regulator of the PI3K/AKT signaling pathway. In addition, accumulating evidence shows that the PTEN/PI3K/AKT/ FOXO3 pathway is responsible for chemotherapy-induced POI [25] and that chronic stress and bisphenol $A$ are related to pathway modulation $[26,27]$. Numerous inhibitors and activators directed against either individual or multiple components of this pathway have already been developed, based on the primary importance of modulating this pathway for cancer treatment (Figure 1) [28]. Currently, several PI3K/AKT/mammalian target of rapamycin (mTOR) modulating agents, primarily agents to induce pathway inhibition, have been identified. LY294002 and wortmannin, which are the best characterized inhibitors, prevent ATP binding and activation of PI3K. Both induce apoptosis of cancer cells and rescue drug sensitivity [29,30]. Perifosine is a water-soluble synthetic alkylphosphocholine with oral bioavailability, and this agent inhibits AKT, reduces cell proliferation, and induces apoptosis in a wide variety of cancers [31]. Rapamycin is an mTOR inhibitor that results in inhibition of proliferation and sensi-

Table 1. Core Hippo signaling pathway in Drosophila and human

\begin{tabular}{ll}
\hline Drosophila & \multicolumn{1}{c}{ Human } \\
\hline Ste20-like protein kinase Hippo (Hpo) & MST1-2 \\
WW domain-containing protein Salvador (Sav), & SAV1/WW45 \\
NDR family protein kinase Warts (Wts) & LATS1-2 \\
Adaptor protein Mob as tumor suppressor (Mats) & MOBKL1A-B \\
Yorkie (Yki) & YAP,TAZ \\
\hline
\end{tabular}

NDR, nuclear Dbf3-related; MST1-2, mammalian sterile 20-like kinase-1; SAV1, protein salvador homolog 1; LATS1-2, large tumor suppressor kinase 1-2; MOBKL1A-B, Mps one binder kinase activator-like 1A-B; YAP, yes-associated protein; TAZ, YAP and transcriptional coactivator with PDZ-binding motif. tization to chemotherapeutic agents, and a recent study suggested that rapamycin can inhibit the excessive activation of primordial follicles in PTEN-knockout rat ovaries, reducing follicle consumption and maintaining ovarian reserve [32]. It has also been suggested that administration of PI3K/AKT/mTOR inhibitors can give rise to potentially life-threatening adverse events, such as pneumonitis and others [33]. While these PI3K/AKT/mTOR inhibitors have been developed for therapeutic use, very few PI3k/AKT/mTOR activators have been identified, since activation of this signaling pathway has been demonstrated to induce malignancies, be associated with poor prognosis, and enhance drug resistance after cancer treatment [28]. Nonetheless, PTEN inhibitors, such as bisperoxovanadium (HOpic), bpV (HOpic), have been shown to effectively activate primordial follicles without any known side effects.

\section{2) Hippo signaling pathway}

First discovered in genetic screens for tumor suppressors in Drosophila, the Hippo signaling pathway is a conserved regulator of organ size, halting organ growth at the appropriate time during development and regeneration [34]. Central to this pathway is a kinase cascade that leads from the tumor suppressor Hippo (MST1 and MST2 in mammals) to the oncoprotein Yorkie (yes-associated protein [YAP] transcriptional coactivator with PDZ-binding motif [TAZ] in mammals), which is a transcriptional coactivator of target genes involved in cell proliferation and survival (Table 1). Activation of YAP/TAZ signaling results in increased expression of intercellular signaling protein (CCN; CYR61, connective tissue growth factor [CTGF], and

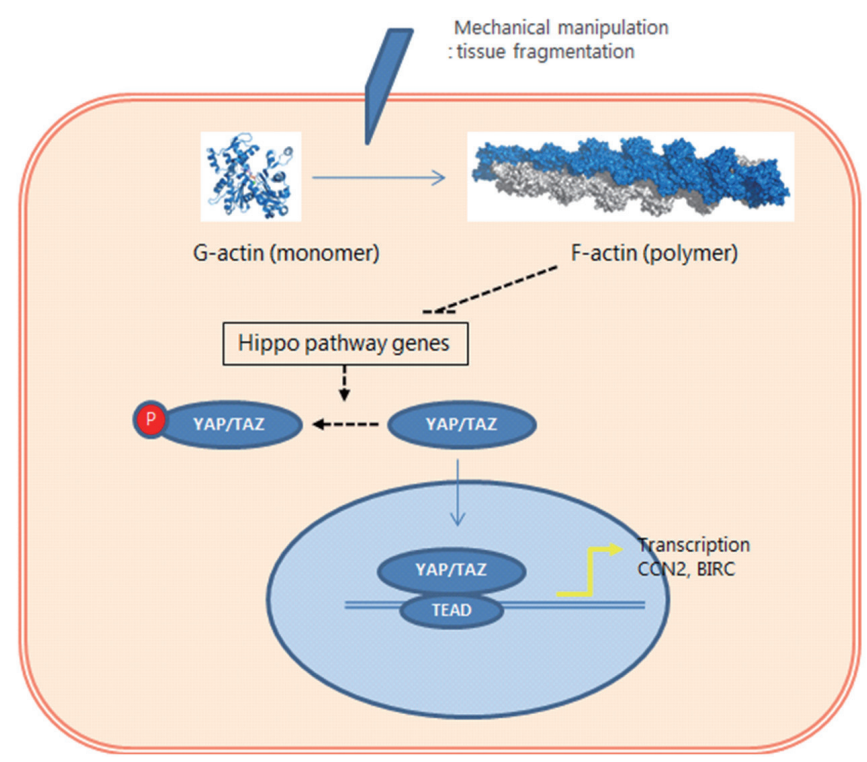

Figure 2. Hippo signaling activation after actin polymerization. $P$, phosphate group; TEAD, TEA domain family member; BIRC, baculoviral inhibitors of apoptosis repeat containing. 
nephroblastoma overexpressed [NOV] proteins) [35]. The Hippo pathway, however, actively prevents aberrant tissue growth by deactivating YAP/TAZ and other transcription factors via phosphorylation.

Dysregulation of Hippo signaling leads to development of various human diseases, including cancer development. In the ovary, Hippo signaling specifically prevents activation of primordial follicles. Mechanical manipulation of ovarian tissue by cutting this tissue into cubes can disrupt this pathway (Figure 2), as ovarian fragmentation changes intercellular tension and facilitates conversion of globular actin (monomer) to fibrous actin (polymer) and this actin polymerization process disrupts Hippo pathway-related genes. Decreasing phospho-YAP ( $p Y A P$ ) levels in concert with increased nuclear localization of YAP leads to increased CCN growth factors and baculoviral inhibitors of apoptosis repeat containing apoptosis inhibitors, which promote follicle growth [35-37].

\section{Murine studies of primordial follicle activation}

Several murine models have demonstrated successful application of AKT stimulation drugs and Hippo signaling disruption on primordial follicle activation [35-38]. Adhikari et al. [36] treated neonatal mouse ovaries with the PTEN inhibitor bpV (HOpic) to generate mature oocytes and confirmed its safety by monitoring the second generation of progeny. Li et al. [37] administered a combination treatment of bpV and 740YP, a PI3K activator, and reported the live birth of healthy pups from cotreated juvenile ovaries transplanted into adult hosts. Kawamura et al. [38] also showed successful live births of mouse pups after allotransplantation of mature follicles activated by fragmentation of day 10 mouse ovaries followed by treatment with a combination of PTEN inhibitor and PI3K activation. Similarly, the same experimental animals underwent xenotransplantation with human ovarian cortical tissue. In this study, human cortical strips that contained secondary and smaller follicles were incubated with AKT stimulators and engrafted into immunodeficient mice. Within 4 weeks, antral follicles were detected, demonstrating rapid follicle growth. These studies suggest the possibility for clinical application of PI3K/PTEN signaling modulation in patients with diminished ovarian reserve.

\section{Clinical application of primordial follicle activation}

Recently, primordial follicle activation technology has been successfully translated for human patients, and estimates suggest that more than 10 live births have occurred (Table 2) [38-41]. The first clinical protocol suggested by Kawamura and colleagues was IVA. For this procedure, $\mathrm{POI}$ patients underwent laparoscopic oophorectomy, and harvested ovarian cortical tissue containing residual follicles was dissected and cut into small strips $\left(1 \times 1 \mathrm{~cm}^{2}, 1-2-\mathrm{mm}\right.$ thickness). Using $10 \%$ of each ovarian strip, the presence of residual follicles was detected by histologic analysis, while the remaining tissue strips were cryopreserved by a vitrification technique. Ovarian cortical strips with confirmed presence of residual follicles were than thawed and further fragmented into 1-2-mm ${ }^{2}$ cubes before IVA drug treatment with $30 \mathrm{mM} \mathrm{bpV}$, a PTEN enzyme inhibitor, and $150 \mathrm{mg} / \mathrm{mL}$

Table 2. Previously reported cases with primordial follicle activation in POI

\begin{tabular}{|c|c|c|c|c|c|c|c|c|}
\hline Study & $\begin{array}{l}\text { Procedure } \\
\text { type }\end{array}$ & $\begin{array}{c}\text { No. of } \\
\text { patients }\end{array}$ & $\begin{array}{l}\text { Inclusion } \\
\text { criteria }\end{array}$ & $\begin{array}{c}\text { Residual } \\
\text { follicle } \\
\text { visualized/total }\end{array}$ & $\begin{array}{l}\text { Follicle } \\
\text { growth/ } \\
\text { total }\end{array}$ & $\begin{array}{l}\text { Mature } \\
\text { oocyte/ } \\
\text { total }\end{array}$ & $\begin{array}{l}\text { Pregnancy/ } \\
\text { total (method) }\end{array}$ & $\begin{array}{l}\text { Live birth } \\
\text { rate }\end{array}$ \\
\hline Kawamura et al. [38] & IVA & 27 & $\begin{array}{l}\text { POI } \\
\text { Amenorrhea }>1 \mathrm{yr} \\
\text { FSH }>40 \mathrm{mlU} / \mathrm{mL}\end{array}$ & $13 / 27$ & $8 / 27$ & $5 / 27$ & 2/27 (IVF) & $1: 27$ \\
\hline Suzuki et al. [39] & IVA & 10 & $\begin{array}{l}\text { POI } \\
\text { Amenorrhea }>4 \mathrm{mo} \\
\text { FSH }>35 \mathrm{mlU} / \mathrm{mL}\end{array}$ & $7 / 10$ & $1 / 10$ & $1 / 10$ & $1 / 10$ (IVF) & $1: 10$ \\
\hline Zhai et al. [40] & $\begin{array}{l}\text { IVA with fresh } \\
\text { tissue transfer }\end{array}$ & 14 & $\begin{array}{l}\text { POI } \\
\text { Amenorrhea > } 1 \mathrm{yr} \\
\text { FSH > } 35 \mathrm{mlU} / \mathrm{mL}\end{array}$ & $7 / 14$ & $6 / 14$ & $4 / 14$ & 1/14 (IVF) & $1: 14$ \\
\hline Fabregues et al. [41] & IVA with drug free & 1 & $\begin{array}{l}\text { POI } \\
\text { Amenorrhea > } 2 \mathrm{yr} \\
\text { FSH } 89.9 \mathrm{mlU} / \mathrm{mL}\end{array}$ & $1 / 1$ & 3/Unknown & 2/Unknown & 1/1 (IVF) & $\begin{array}{r}24 \text { Weeks } \\
\text { ongoing }\end{array}$ \\
\hline
\end{tabular}

POI, primary ovarian insufficiency; IVA, in vitro activation; FSH, follicle-stimulating hormone; IVF, in vitro fertilization; OFFA, ovarian fragmentation for follicular activation.

a) Unofficial data are from conference presentations of stated scientists. 
740YP, a PI3K stimulator, for 24 hours followed by incubation with 740YP alone for another 24 hours. Then, the ovarian fragments were thoroughly washed with warmed cultured medium and auto-transplanted beneath the serosa of fallopian tubes. A total of $27 \mathrm{POI}$ patients were initially recruited for laparoscopic oophorectomy, resulting in two pregnancies and 1 healthy delivery [39]. More recently, Zhai et al. [40] reported a single successful delivery after simplified IVA by grafting fresh ovarian tissue, and Fabregues et al. [41] reported another successful delivery without the use of PTEN/PI3K/AKT/ FOXO3 signaling modulators.

As mentioned for murine studies, fragmentation of ovarian tissues activates the primordial follicles even in the absence of further signaling modulation, and thus, OFFA has been used for POI treatment. This protocol excludes IVA drug treatment. Together with infusion of bone marrow-derived stem cells into ovarian artery, Pellicer et al. (Spain) reported three natural pregnancies in POI patients; however, these results have not been officially published.

\section{Limitations and further applications for primordial follicle activation}

While IVA offers promise for POI patients, its success rate has presented a challenge. Considering that natural pregnancy rate after long-term follow-up of $\mathrm{POI}$ patients receiving hormone replacement therapy reaches approximately $1.5 \%-5 \%$, pregnancy rate after IVA is relatively disappointing [42]. However, the pregnancy rate following in vitro fertilization in its early days only reached $9 \%$. Thus, while IVA technology is innovative, there is still considerable room for clinical improvement.

Another concern following IVA is the possibility of carcinogenic or other deleterious effects of IVA drugs. The PI3K/AKT pathway is frequently activated in human cancers, and although no cancer development following IVA has been reported, we should remain cautious about its clinical application due to the ability of the synthetic IVA drugs to modulate a well-known molecular cancer pathway. Additionally, a recent study suggested that inhibition of PTEN via a synthetic inhibitor may result in activation of primordial follicles but may compromise the development of the growing follicle [43]. In addition to the risks of the IVA drugs, there are other limitations of this technique. IVA technology involves at least one laparoscopic surgery, which is fairly extensive. Epigenetic studies of embryos and the health of the baby following IVA have yet to be confirmed. Thus, future endeavors should investigate safer and more effective methods for IVA and discover additional physiologic candidates for the modulation of the PTEN/PI3K/AKT/FOXO3 signaling pathway. In this respect, recent success regarding complete in vitro development of oocytes derived from human ovarian tissue that contained primordial/ unilaminar follicles is quite encouraging. Until recently, a culture system that could support complete growth from the earliest stages of human follicle development through metaphase II had not been reported. McLaughlin et al. [44] however, recently described such a four-step culture system. In this report, full in vitro culture of the follicle took only 21 days, which is extremely fast compared to in vivo systems. Confocal microscopy analysis of those oocytes revealed abnormally large polar bodies, suggesting that further optimization remains to be achieved [44]. In the future, combining IVA with a complete in vitro culture system to achieve pregnancy may abrogate the need for surgery and minimize the risk for cancer development.

In addition to hope for POI patients, IVA offers a promising option for fertility preservation in cancer patients and prepubertal girls. For patients who are not candidates for oocyte and embryo cryopreservation, the use of IVA to activate primordial follicles may increase the effectiveness of ovarian tissue freezing by maximizing the number of oocytes available [45]. Additionally, the ability to recruit these dormant follicles into the pool of activated follicles and support their complete development in vitro would help to obtain a greater number of competent oocytes for assisted reproduction techniques, thus improving the chance of pregnancy especially for poor responders [46].

\section{Conclusion}

IVA represents innovative technology for $\mathrm{POI}$ patients. Based on primordial follicle activation using tissue fragmentation and PTEN/PI3K/ AKT/FOXO3 signaling modulation, this technique has been successfully applied for POI patients, resulting in healthy deliveries. Furthermore, IVA may provide a promising option for fertility preservation in cancer patients and prepubertal girls, whose fertility preservation choices are limited to tissue cryopreservation. The use of IVA as a treatment for POI remains highly experimental with limited data on outcomes and a requirement for invasive procedures. Additionally, low pregnancy rates and possible carcinogenic effects following IVA require special caution. Thus, IVA should be performed only under approval of an Institutional Review Board to ensure patient safety. In the future, a better understanding of the mechanism underlying primordial follicle activation, improvements in culture and tissue transplantation technology, and development of safer physiologic IVA drugs may help to optimize this new technology, yielding better clinical outcomes for patients.

\section{Conflict of interest}

No potential conflict of interest relevant to this article was reported. 


\section{ORCID}

Hye Nam Lee https://orcid.org/0000-0002-8427-4150

Eun Mi Chang https://orcid.org/0000-0001-9256-5057

\section{Author contributions}

Conceptualization, Data curation, Funding acquisition, Formal analysis, Methodology, Project administration, \& Visualization: EMC. Writing - original draft: HNL, EMC. Writing - review \& editing: HNL, EMC.

\section{References}

1. De Vos M, Devroey P, Fauser BC. Primary ovarian insufficiency. Lancet 2010;376:911-21.

2. Coulam CB, Adamson SC, Annegers JF. Incidence of premature ovarian failure. Obstet Gynecol 1986;67:604-6.

3. Nelson LM. Clinical practice: primary ovarian insufficiency. N Engl J Med 2009;360:606-14.

4. European Society for Human Reproduction and Embryology (ESHRE) Guideline Group on POI, Webber L, Davies M, Anderson R, Bartlett J, Braat D, et al. ESHRE guideline: management of women with premature ovarian insufficiency. Hum Reprod 2016;31: 926-37.

5. Cordts EB, Christofolini DM, Dos Santos AA, Bianco B, Barbosa CP. Genetic aspects of premature ovarian failure: a literature review. Arch Gynecol Obstet 2011;283:635-43.

6. Haller-Kikkatalo K, Uibo R, Kurg A, Salumets A. The prevalence and phenotypic characteristics of spontaneous premature ovarian failure: a general population registry-based study. Hum Reprod 2015;30:1229-38.

7. Woad KJ, Watkins WJ, Prendergast D, Shelling AN. The genetic basis of premature ovarian failure. Aust N Z J Obstet Gynaecol 2006:46:242-4.

8. McGee EA, Hsueh AJ. Initial and cyclic recruitment of ovarian follicles. Endocr Rev 2000;21:200-14.

9. Skinner MK. Regulation of primordial follicle assembly and development. Hum Reprod Update 2005;11:461-71.

10. Adhikari D, Flohr G, Gorre N, Shen Y, Yang H, Lundin E, et al. Disruption of Tsc2 in oocytes leads to overactivation of the entire pool of primordial follicles. Mol Hum Reprod 2009;15:765-70.

11. Ding X, Zhang X, Mu Y, Li Y, Hao J. Effects of BMP4/SMAD signaling pathway on mouse primordial follicle growth and survival via up-regulation of Sohlh2 and c-kit. Mol Reprod Dev 2013; 80:70-8.

12. Lee WS, Otsuka F, Moore RK, Shimasaki S. Effect of bone morphogenetic protein-7 on folliculogenesis and ovulation in the rat. Biol Reprod 2001;65:994-9.

13. Tang K, Yang WC, Li X, Wu CJ, Sang L, Yang LG. GDF-9 and bFGF enhance the effect of FSH on the survival, activation, and growth of cattle primordial follicles. Anim Reprod Sci 2012;131: 129-34.

14. Huang EJ, Manova K, Packer Al, Sanchez S, Bachvarova RF, Besmer P. The murine steel panda mutation affects kit ligand expression and growth of early ovarian follicles. Dev Biol 1993;157: 100-9.

15. Yoshida H, Takakura N, Kataoka H, Kunisada T, Okamura H, Nishikawa SI. Stepwise requirement of c-kit tyrosine kinase in mouse ovarian follicle development. Dev Biol 1997;184:122-37.

16. Kezele PR, Nilsson EE, Skinner MK. Insulin but not insulin-like growth factor-1 promotes the primordial to primary follicle transition. Mol Cell Endocrinol 2002;192:37-43.

17. Nilsson EE, Larsen G, Skinner MK. Roles of Gremlin 1 and Gremlin 2 in regulating ovarian primordial to primary follicle transition. Reproduction 2014;147:865-74.

18. Nilsson EE, Kezele P, Skinner MK. Leukemia inhibitory factor (LIF) promotes the primordial to primary follicle transition in rat ovaries. Mol Cell Endocrinol 2002;188:65-73.

19. Durlinger AL, Kramer P, Karels B, de Jong FH, Uilenbroek JT, Grootegoed JA, et al. Control of primordial follicle recruitment by anti-Mullerian hormone in the mouse ovary. Endocrinology 1999;140:5789-96.

20. Bonnet A, Cabau C, Bouchez O, Sarry J, Marsaud N, Foissac S, et al. An overview of gene expression dynamics during early ovarian folliculogenesis: specificity of follicular compartments and bidirectional dialog. BMC Genomics 2013;14:904.

21. Hosaka T, Biggs WH 3rd, Tieu D, Boyer AD, Varki NM, Cavenee WK, et al. Disruption of forkhead transcription factor (FOXO) family members in mice reveals their functional diversification. Proc Natl Acad Sci U S A 2004;101:2975-80.

22. Castrillon DH, Miao L, Kollipara R, Horner JW, DePinho RA. Suppression of ovarian follicle activation in mice by the transcription factor Foxo3a. Science 2003;301:215-8.

23. Reddy P, Liu L, Adhikari D, Jagarlamudi K, Rajareddy S, Shen Y, et al. Oocyte-specific deletion of Pten causes premature activation of the primordial follicle pool. Science 2008;319:611-3.

24. John GB, Gallardo TD, Shirley LJ, Castrillon DH. Foxo3 is a PI3Kdependent molecular switch controlling the initiation of oocyte growth. Dev Biol 2008;321:197-204.

25. Chang EM, Lim E, Yoon S, Jeong K, Bae S, Lee DR, et al. Cisplatin induces overactivation of the dormant primordial follicle through PTEN/AKT/FOXO3a pathway which leads to loss of ovarian reserve in mice. PLoS One 2015;10:e0144245.

26. Xu M, Sun J, Wang Q, Zhang Q, Wei C, Lai D. Chronic restraint 
stress induces excessive activation of primordial follicles in mice ovaries. PLoS One 2018;13:e0194894.

27. Hu Y, Yuan DZ, Wu Y, Yu LL, Xu LZ, Yue LM, et al. Bisphenol A initiates excessive premature activation of primordial follicles in mouse ovaries via the PTEN signaling pathway. Reprod Sci 2018; 25:609-20.

28. Matsuda S, Nakanishi A, Wada Y, Kitagishi Y. Roles of PI3K/AKT/ PTEN pathway as a target for pharmaceutical therapy. Open Med Chem J 2013;7:23-9.

29. Imai Y, Yamagishi H, Ono Y, Ueda Y. Versatile inhibitory effects of the flavonoid-derived PI3K/Akt inhibitor, LY294002, on ATPbinding cassette transporters that characterize stem cells. Clin Transl Med 2012;1:24.

30. Gross ER, Peart JN, Hsu AK, Auchampach JA, Gross GJ. Extending the cardioprotective window using a novel delta-opioid agonist fentanyl isothiocyanate via the PI3-kinase pathway. Am J Physiol Heart Circ Physiol 2005;288:H2744-9.

31. Gills JJ, Dennis PA. Perifosine: update on a novel Akt inhibitor. Curr Oncol Rep 2009;11:102-10.

32. Guo Y, Kwiatkowski DJ. Equivalent benefit of rapamycin and a potent mTOR ATP-competitive inhibitor, MLN0128 (INK128), in a mouse model of tuberous sclerosis. Mol Cancer Res 2013;11:46773.

33. Albiges L, Chamming's F, Duclos B, Stern M, Motzer RJ, Ravaud A, et al. Incidence and management of mTOR inhibitor-associated pneumonitis in patients with metastatic renal cell carcinoma. Ann Oncol 2012;23:1943-53.

34. Pan D. The hippo signaling pathway in development and cancer. Dev Cell 2010;19:491-505.

35. Cheng Y, Feng Y, Jansson L, Sato Y, Deguchi M, Kawamura K, et al. Actin polymerization-enhancing drugs promote ovarian follicle growth mediated by the Hippo signaling effector YAP. FASEB J 2015;29:2423-30.

36. Adhikari D, Gorre N, Risal S, Zhao Z, Zhang H, Shen Y, et al. The safe use of a PTEN inhibitor for the activation of dormant mouse primordial follicles and generation of fertilizable eggs. PLoS One 2012;7:e39034.

37. Li J, Kawamura K, Cheng Y, Liu S, Klein C, Liu S, et al. Activation of dormant ovarian follicles to generate mature eggs. Proc Natl Acad Sci U S A 2010;107:10280-4.

38. Kawamura K, Cheng Y, Suzuki N, Deguchi M, Sato Y, Takae S, et al. Hippo signaling disruption and Akt stimulation of ovarian follicles for infertility treatment. Proc Natl Acad Sci U S A 2013;110: 17474-9.

39. Suzuki N, Yoshioka N, Takae S, Sugishita Y, Tamura M, Hashimoto $S$, et al. Successful fertility preservation following ovarian tissue vitrification in patients with primary ovarian insufficiency. Hum Reprod 2015;30:608-15.

40. Zhai J, Yao G, Dong F, Bu Z, Cheng Y, Sato Y, et al. In vitro activation of follicles and fresh tissue auto-transplantation in primary ovarian insufficiency patients. J Clin Endocrinol Metab 2016;101: 4405-12.

41. Fabregues F, Ferreri J, Calafell JM, Moreno V, Borras A, Manau D, et al. Pregnancy after drug-free in vitro activation of follicles and fresh tissue autotransplantation in primary ovarian insufficiency patient: a case report and literature review. J Ovarian Res 2018; 11:76.

42. Check JH, Wilson C, DiAntonio G, DiAntonio A. In vitro fertilization (IVF) outcome in women in overt menopause attempting to induce follicular maturation by follicle stimulating hormone (FSH) receptor down-regulation. Clin Exp Obstet Gynecol 2016; 43:181-3.

43. McLaughlin M, Kinnell HL, Anderson RA, Telfer EE. Inhibition of phosphatase and tensin homologue (PTEN) in human ovary in vitro results in increased activation of primordial follicles but compromises development of growing follicles. Mol Hum Reprod 2014;20:736-44.

44. McLaughlin M, Albertini DF, Wallace WH, Anderson RA, Telfer EE. Metaphase II oocytes from human unilaminar follicles grown in a multi-step culture system. Mol Hum Reprod 2018;24:135-42.

45. Resetkova N, Hayashi M, Kolp LA, Christianson MS. Fertility preservation for prepubertal girls: update and current challenges. Curr Obstet Gynecol Rep 2013;2:218-25.

46. Kallen A, Polotsky AJ, Johnson J. Untapped reserves: controlling primordial follicle growth activation. Trends Mol Med 2018;24: 319-31. 